\title{
Good Clinical Success Rates Are Seen 5 Years After Meniscal Repair in Patients Regularly Undertaking Extreme Flexion
}

\author{
Jalal Odeh, M.D., Sultan al Maskari, F.R.C.S.Ed.(T\&O), Sameer Raniga, F.R.C.R., \\ Mahmood al Hinai, B.Sc., Alok Mittal, D.N.B., and Ahmed al Ghaithi, M.D.
}

\begin{abstract}
Purpose: To report the functional and radiologic outcomes of meniscal repair healing in a cohort of patients with a high demand for loaded extreme flexion angles after undergoing meniscal repair. Methods: We performed a retrospective clinical and radiologic evaluation of patients who perform extreme knee flexion activities on a daily basis at a minimum follow-up of 2 years after meniscal repair. International Knee Documentation Committee, Lysholm, and Tegner scores were obtained, and clinical examinations and radiologic (magnetic resonance imaging and radiography) evaluations were performed. Results: Of 47 eligible patients, 39 patients (40 knees) were available for review with an average follow-up time of 5 years (range, 2-9 years). The average age was 26.7 years (range, 19-39 years); 38 patients were men. The average time from injury to surgery was 20.9 months (range, 3 days to 120 months). Associated anterior cruciate ligament injury was present in 31 knees, but only 20 underwent simultaneous anterior cruciate ligament surgery. The mean International Knee Documentation Committee score was 88.9 (range, 53-99). The mean Lysholm score was 90.9 (range, 48-100). The mean Tegner activity level dropped from 6.18 before injury to 5.51 at the time of evaluation. According to the Barrett criteria for clinical outcomes, complete healing was observed in 29 of 40 knees $(72.5 \%)$. There was a statistically significant correlation between the functional outcomes and the clinical outcomes $(P=.008)$. On magnetic resonance imaging, 22 of $38 \mathrm{knees}$ $(57.9 \%)$ showed completely healed menisci. Conclusions: The results of this study suggest that loaded deep knee flexion may be safe after a period of restricted rehabilitation, and clinical and radiologic tissue healing is independent of the overall functional outcome. Level of Evidence: Level IV, case series with subgroup analysis.
\end{abstract}

M eniscal tears are common knee injuries in active youth. ${ }^{1}$ Loss of the meniscal functional unit is thought to lead to progressive arthrosis, whereas its preservation seems to slow this process. ${ }^{2-4} \mathrm{~A}$ better understanding of the anatomy and pathophysiology of

From Orthopaedic Training Programme, Oman Medical Specialties Board, Al Azaiba, Sultanate of Oman (J.O.); Division of Orthopaedic Surgery, Sultan Qaboos University Hospital, Al Khod, Sultanate of Oman (S.a.M., A.a.G.); Department of Radiology, Sultan Qaboos University Hospital, Al Khod, Sultanate of Oman (S.R., A.M.); and Department of Physiotherapy, Sultan Qaboos University Hospital, Al Khod, Sultanate of Oman (M.a.H.).

The authors report no conflicts of interest in the authorship and publication of this article. Full ICMJE author disclosure forms are available for this article online, as supplementary material.

Received February 14, 2021; accepted August 24, 2021.

Address correspondence to Jalal Odeh, M.D., Orthopaedic Training Programme, Oman Medical Specialties Board, PO Box 1948, PC 130, Al Azaiba, Muscat, Sultanate of Oman.E-mail:dr.jalal3@gmail.com

(C) 2021 THE AUTHORS. Published by Elsevier Inc. on behalf of the Arthroscopy Association of North America. This is an open access article under the CC BY-NC-ND license (http://creativecommons.org/licenses/by-nc-nd/4.0/).

2666-061X/21182

https://doi.org/10.1016/j.asmr.2021.08.011 the meniscus and greater awareness of the deleterious effects of meniscectomy have led to calls for tissue preservation to prevent or delay the onset of knee arthrosis. $^{1,5}$ Improvements in training, arthroscopic technology, and surgical techniques, as well as greater options of suture devices, have made meniscal repair a much simpler task.

Comprehensive management of meniscal tears should be based on the pathoanatomic features of the tear as well as the functional demands of the patient. The literature is replete with information relating to the effects of meniscal tear type, repair site, and patient age on the success of repair. ${ }^{6-9}$ On the other hand, there is a lack of basic information concerning the knee position's impact during various daily activities on meniscal repair and healing. Deep flexion movements beyond $90^{\circ}$ (e.g., squatting and kneeling) are important movements for daily living activities and are common among many populations. ${ }^{10}$ The high angle of flexion results in greater loads on the posterior parts of the meniscus and can influence its healing. ${ }^{6}$ Netravali et al. ${ }^{1}$ studied mechanical loading during activities 

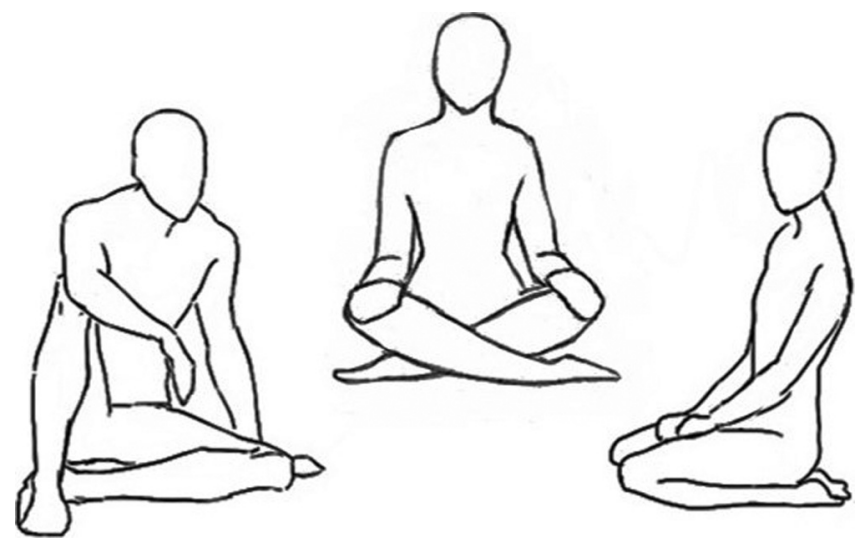

Fig 1. Demonstration of deep knee flexion positions necessary in activities of daily living of study group.

requiring deep flexion and showed its influence on pathologic joint changes. They emphasized the importance of considering the magnitude of the forces generated during deep flexion activities when performing meniscal repair procedures. Despite this, there is little published evidence on the clinical effects of deep knee flexion after meniscal repair.

Repairs of meniscal tears are frequently performed along with other knee procedures such as anterior cruciate ligament reconstruction (ACLR). Immediate range of motion (ROM) after meniscal repair is considered an import factor in rehabilitation. Spang et al. ${ }^{11}$ reviewed the biomechanical and biological aspects of existing evidence regarding ROM restrictions after meniscal repair, and they found that the quality of evidence is low, with no consensus regarding how much knee flexion can be allowed. Lin et al. ${ }^{12}$ simulated open-chain exercises at high flexion angles and studied their effect on meniscal repair separation. They concluded that nonrestrictive, resistance-free open-chain motion protocols do not place undue stress on the repaired meniscus.

Asian populations spend a significant length of time, frequently during the day, squatting and kneeling for social and religious activities. ${ }^{13}$ These activities load the knee in full flexion accompanied by extreme internal or external rotation exerting high shear and torsional forces on the joint structures (Fig 1). ${ }^{10}$ The purpose of this study was to report the functional and radiologic outcomes of meniscal repair healing in a cohort of patients with a high demand for loaded extreme flexion angles after undergoing meniscal repair. We hypothesized that regular extreme knee flexion would adversely affect the medium-term survivorship and functional outcomes of meniscal repair.

\section{Methods}

After institutional review board (reference No. SQUEC/119/17), eligible patients were called for evaluation. Target patients who underwent surgery between January 1, 2009, and June 30, 2018, were identified from
Table 1. Inclusion and Exclusion Criteria

Inclusion Criteria

Age $<40$ yr at time of surgery

Both sexes

Meniscal tear involving posterior horn of either meniscus

Minimum follow-up period of $2 \mathrm{yr}$; no or mild degenerative changes at time of surgery

Must squat and sit on floor with knee in full flexion on regular basis for daily total of $\geq 2 \mathrm{~h}$ (refer to Fig 2), with screening of patients using the following questions:

Do you eat $\geq 2$ meals while sitting on the floor?

Do you sit on the floor to read or play card or board games?

Do you conduct kneeling prayer on the floor for a daily total of $\geq \mathrm{lh}$ ?

Are you able to use an Eastern-style toilet?

Exclusion criteria

Age $\geq 40$ yr at time of surgery

Does not squat and sit on floor with knee in full flexion on regular basis for daily total of $\geq 2 \mathrm{~h}$

Tears not involving posterior horns of menisci

Meniscal root tears

Underwent surgery on same knee after meniscal repair

Trauma or injury to same limb after meniscal repair needing medical intervention

Moderate or advanced degenerative changes at time of surgery

Follow-up period $<2 \mathrm{yr}$

Patient not willing to participate in study

hospital records for this retrospective study. The inclusion and exclusion criteria are summarized in Table 1.

\section{Surgical Technique}

All surgical procedures were performed under tourniquet control by 1 surgeon (S.a.M.) in 2 centers: a university teaching hospital and a private hospital. Meniscal repair was performed using nonabsorbable sutures by the all-inside method with the Meniscal Cinch (Arthrex, Naples, FL) for the posterior horn in all knees. In addition, inside-out (9 knees) and outside-in (6 knees) techniques were used. No bioactivating adjuncts, such as platelet-rich plasma, venous blood clots, or bone marrow stimulation, were used. The quadrupled hamstring tendon autograft technique (GraftLink all-inside technique; Arthrex) was used when ACLR was performed. A few fresh proximal anterior cruciate ligament (ACL) tears underwent primary repair.

\section{Rehabilitation Protocol}

Postoperatively, knees were protected with a hinged brace (6 weeks for isolated repairs and 3 months for ACLR) and patients were instructed to ambulate with minimal weight bearing using crutches and to avoid knee flexion beyond $90^{\circ}$ for 6 weeks. They were advised to return to normal socioreligious activities 6 months after surgery.

\section{Evaluation Details}

After informed written consent was obtained, patients were assessed as follows: patient-reported outcome 


\begin{tabular}{|l|l|}
\hline $\begin{array}{l}671 \text { knee } \\
\text { arthroscopic } \\
\text { procedures }\end{array}$ & $\longrightarrow \begin{array}{l}551 \text { Excluded: } \\
\text { No meniscal } \\
\text { repair }\end{array}$ \\
\hline
\end{tabular}

Fig 2. Filtration flow diagram showing patient selection process and surgical procedures performed in patients. (ACL, anterior cruciate ligament; ACLR, anterior cruciate ligament reconstruction; OA, osteoarthritic.)
Meniscal repair 120

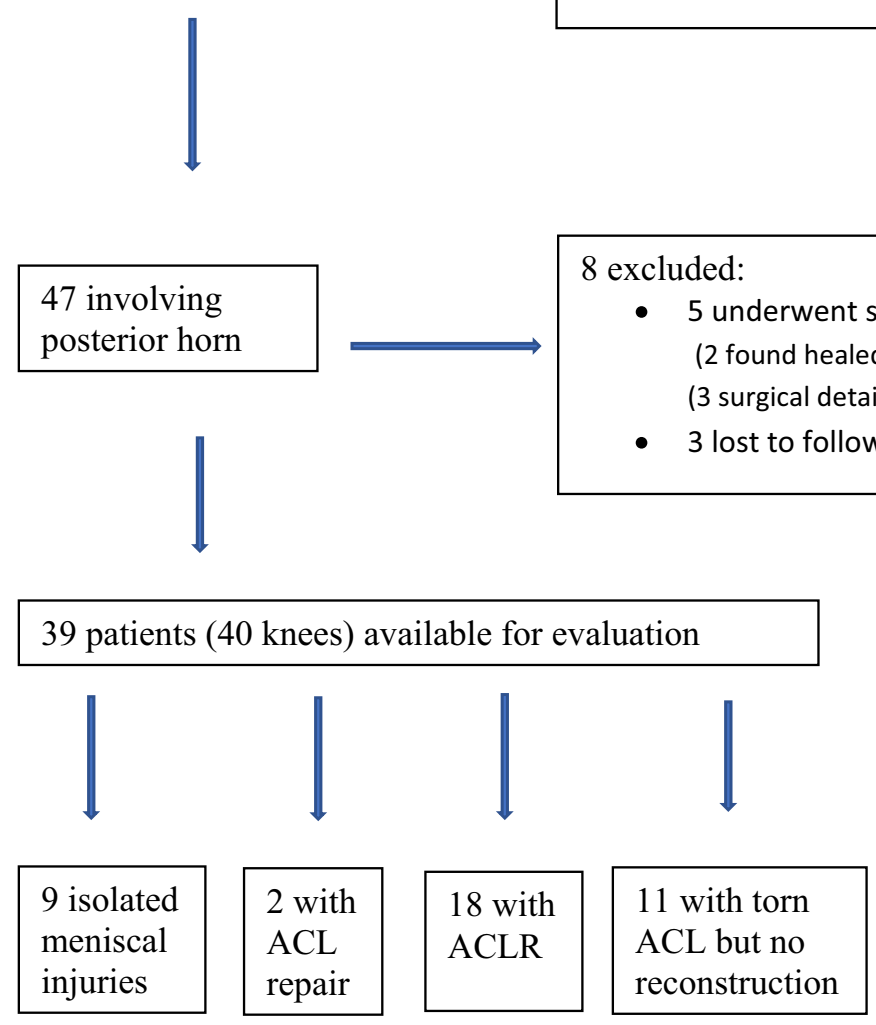

73 Excluded:

- Older than 40 years

- Don't do daily deep flexion

- OA changes

- Posterior horn not involved scores (subjective International Knee Documentation Committee [IKDC] score, Lysholm score, and Tegner activity level scale) were recorded. The clinical criteria of Barrett et al. ${ }^{14}$ were used to evaluate clinical healing, and magnetic resonance imaging (MRI) scans and radiographs were used to evaluate radiologic healing and outcomes. ${ }^{15}$ MRI signals at the repair site were assessed on T2-weighted images using the signal characteristics of the grading systems of Kijowski et al. ${ }^{16}$ and Crues et al. ${ }^{17}$ Degenerative changes on weight-bearing plain radiographs were graded using the Kellgren-Lawrence grading system. ${ }^{18}$

A trained clinician (J.O.) (other than the operating surgeon) and a qualified musculoskeletal physiotherapist
(M.a.H.) independently obtained the outcome scores and carried out the clinical examinations. Two musculoskeletal radiologists (S.R. and A.M.) reported the findings of the MRI scans and radiographs in consensus.

\section{MRI Protocol}

MRI scanning of the knee joint was performed on a 1.5-T scanner (Siemens, Erlangen, Germany) using a phased-array knee coil. After 3 plane localizers were obtained, multiplanar images were obtained using the following sequences: proton density-weighted fatsuppressed turbo spin echo in the transverse, sagittal, and coronal planes; $\mathrm{Tl}$ turbo spin echo in the coronal plane; and turbo inversion recovery magnitude in the 
Table 2. Patient Demographic Characteristics and Injury Patterns

\begin{tabular}{lc}
\hline \multicolumn{1}{c}{ Variable } & Data \\
\hline Sex & 38 \\
Male & 1 \\
Female & \\
Age, yr & 26.7 \\
Mean & $18-39$ \\
Range & \\
Knee & 22 \\
Right & 18 \\
Left & \\
Side & 26 \\
Medial & 9 \\
Lateral & 5 \\
Both & \\
Tear & \\
Bucket handle & 12 \\
CH & 3 \\
NCH & \\
Radial & 6 \\
CH & 1 \\
NCH & \\
Horizontal & 8 \\
CH & 3 \\
NCH & \\
Complex & CH \\
NCH & 5 \\
\hline NOTE. Datarepresented ns & 2 \\
\hline
\end{tabular}

NOTE. Data are presented as number of patients (sex) or number of knees unless otherwise indicated.

$\mathrm{CH}$, clinical healing; $\mathrm{NCH}$, no clinical healing.

sagittal plane. All the images were acquired with a slice thickness of 3 to $4 \mathrm{~mm}$, field of view ranging from 160 to $172 \mathrm{~mm}$, and matrix resolution of at least $256 \times 256$. An arthrogram was not included in the protocol.

The MRI scans were read by 2 senior musculoskeletal radiologists (S.R. and A.M.) in consensus. Both the radiologists were provided with the surgical history and the site of meniscal repair. Preoperative MRI scans were also available for review. The radiologists were not provided with the clinical evaluation details. A retear was diagnosed when the site of repair showed a fluidlike signal extending into the superior or inferior articular surface on T2-weighted images. Other MRI criteria for retear were as follows: (1) change in the meniscal signal pattern with the presence of a new signal abnormality or orientation different from the primary tear pattern on baseline MRI, (2) displaced meniscal fragment, and (3) irregular size and/or contour in the absence of meniscectomy. The absence of a fluid-like signal at the site of repair on proton density or T2-weighted images was considered to indicate a healed meniscus. ${ }^{16,17}$

\section{Statistical Analysis}

The correlation between continuous variables was assessed using the Spearman $\rho$. Comparisons between continuous variables and binary categories were performed with the Mann-Whitney $U$ test. For comparisons between categorical variables, the Fisher exact test was used. The significance level was set to $P \leq .05$. Statistical analysis was performed using SPSS software (version 25; IBM, Armonk, NY).

\section{Results}

A total of 671 arthroscopic knee procedures were performed from January 1, 2009, to June 30, 2018; meniscal repair was performed in a total of 120 patients. Of these patients, 47 met the inclusion criteria, but only 39 patients (40 knees) were available for evaluation (Fig 2). The injury was sports related in all patients. The mean follow-up period was 5 years (range, 2-9 years). Table 2 summarizes the patients' demographic characteristics and injury patterns. Because of delays in presentation to our clinic, the average time from injury to surgery was 20.9 months (range, 3 days to 120 months; mode, 12 months). An isolated meniscal tear was present in 9 knees $(22.5 \%)$. A concomitant ACL injury was present in 31 knees $(77.5 \%)$, of which $18(58.1 \%)$ underwent ACLR and meniscal repair, whereas 2 knees $(6.5 \%)$ with fresh femoral end ACL avulsion underwent both primary ACL and meniscal repairs. Eleven patients (11 knees) declined ACLR and underwent only meniscal repair because either they presented acutely with a locked knee and were not prepared for prolonged ACLR rehabilitation or they were troubled only by locking or pseudo-locking episodes without any instability.

The mean IKDC score was 88.9 (range, 53-99). The mean Lysholm score was 90.9 (range, 48-100), whereby 25 patients $(64.1 \%)$ had excellent results and 9 patients $(23.1 \%)$ had good results. The mean Tegner activity level dropped from 6.18 before injury to 5.51 at the time of evaluation (statistically significant difference, with $P=.001$ ). According to the Barrett criteria for clinical outcomes, complete healing was observed in 29 of 40 knees $(72.5 \%)$. There was a statistically significant correlation between the functional outcomes and the clinical outcomes $(P=.008)$.

Only 38 knee MRI scans were performed because 2 patients did not attend their MRI appointments. On MRI, 22 knees $(57.9 \%)$ had Crues grades 0 to 2 , indicating healed menisci; 16 knees $(42.1 \%)$ had grade 3 and were considered not healed. Plain radiographs were taken of 37 operated knees and showed a mean KL grade of 2 (range, 0-3; mode, 2) compared with 29 radiographs of the nonoperated knees with a mean grade of 1 (range, $0-3$; mode, 1 ).

The clinical (Barrett) and MRI healing rates in the ACLR group were $88.9 \%$ (16 of 18 patients) and $61.1 \%$ (11 of 18 patients), respectively. The clinical (Barrett) and MRI healing rates in the group undergoing only meniscal repair with an ACL injury were $63.6 \%$ (7 of 11 patients) and $36.4 \%$ (4 of 11 patients), respectively. 
Table 3. Associations Between Functional Outcome Scores and Healing Rates

\begin{tabular}{|c|c|c|c|c|}
\hline Clinical Outcome & No. of Knees & Mean & SD & $P$ Value* \\
\hline \multicolumn{5}{|c|}{ Association between clinical and functional outcomes } \\
\hline Lysholm score & & & & .036 \\
\hline Healed & 29 & 93.4 & 6.8 & \\
\hline IKDC score & & & & .008 \\
\hline Healed & 29 & 90.9 & 7.4 & \\
\hline Not healed & 11 & 83.9 & 11.9 & \\
\hline Healed & 22 & 90.5 & 12.2 & \\
\hline Not healed & 16 & 90.2 & 8.6 & \\
\hline IKDC score & & & & .13 \\
\hline Healed & 22 & 89.7 & 9.5 & \\
\hline Not healed & 16 & 86.1 & 9.8 & \\
\hline \multicolumn{5}{|l|}{ Time from injury to surgery, mo } \\
\hline Not healed & 16 & 31.9 & 35.6 & \\
\hline Healed & 22 & 17.7 & 22.2 & \\
\hline
\end{tabular}

IKDC, International Knee Documentation Committee; MRI, magnetic resonance imaging; SD, standard deviation.

*Statistical significance using Mann-Whitney test $(P<.05)$.

Nine patients had an isolated meniscal tear with an intact ACL. The clinical and MRI healing rates for patients with isolated meniscal tears were $66.6 \%$ (6 of 9 patients) and $55.5 \%$ (5 of 9 patients), respectively. The average time from injury to surgery was 20 months in patients who achieved clinical (Barrett) healing compared with 24.8 months in those who did not heal. On MRI, the average time for patients who achieved healing was 17.7 months versus 31.9 months for those who did not heal. Neither of these differences was statistically significant $(P=.508$ and $P=.139$, respectively). Table 3 summarizes the associations between the functional outcome scores and the healing rate (clinical and MRI), as well as the relation to the time interval between injury and surgery.

\section{Discussion}

Our study has shown that good clinical success rates can be obtained at an average of 5 years after meniscal repair in populations demanding loaded extreme knee flexion and rotation, even without consideration of the time gap from injury to surgery. Despite the high flexion demand that creates excessive forces on the menisci's posterior horns and the chronicity of the tears, $87.2 \%$ of our patients reported good or excellent results according to the Lysholm knee score and the average IKDC score was 88.9-findings that are comparable to published results in other populations. ${ }^{8,9,19-21}$ However, these findings do not necessarily indicate complete structural healing of the repair. In a second-look arthroscopic study, Ahn et al. ${ }^{8}$ found that all 17 patients with incompletely healed repairs reported clinically successful results. Recent advances in suturing materials and techniques have allowed applications of complex suture configurations and repair of complex tears, providing stronger and more durable repairs, resulting in greater stability of the repaired tissues. Lin et al. ${ }^{12}$ described deformation of the meniscus during knee flexion in a cadaveric study, which permits the interaction between the femur and meniscus. They concluded that the knee kinematics of a repaired meniscus closely resemble that of an intact meniscus and the rigid repair can tolerate nonrestrictive, resistance-free open-chain ROM protocols. In a meta-analysis of all-inside and inside-out meniscal repair techniques, Kang et al. ${ }^{19}$ found success rates between $70.8 \%$ and $93.3 \%$, concluding that different techniques were equally effective. Although our patients underwent a cautious rehabilitation protocol, we are unable to make any conclusions about the effect of this on our results. Of 18 articles undergoing a literature review, 14 advised a delayed return to sport after meniscal repair by 3 to 6 months. ${ }^{22}$ However, the authors concluded that there is no evidence to suggest that accelerated rehabilitation and an early return to sports jeopardize the success rate of meniscal repair. ${ }^{22}$

The discrepancy between radiologic (MRI) and clinical (Barrett) healing rates $(57.9 \%$ and $72.5 \%$, respectively) is in keeping with the findings of most published studies. In their post-repair MRI and second-look arthroscopic study, Miao et al. ${ }^{23}$ reported that the diagnostic accuracy of all modalities of conventional MRI was below $70 \%$. They also noted that MRI's diagnostic accuracy improved with extended time 


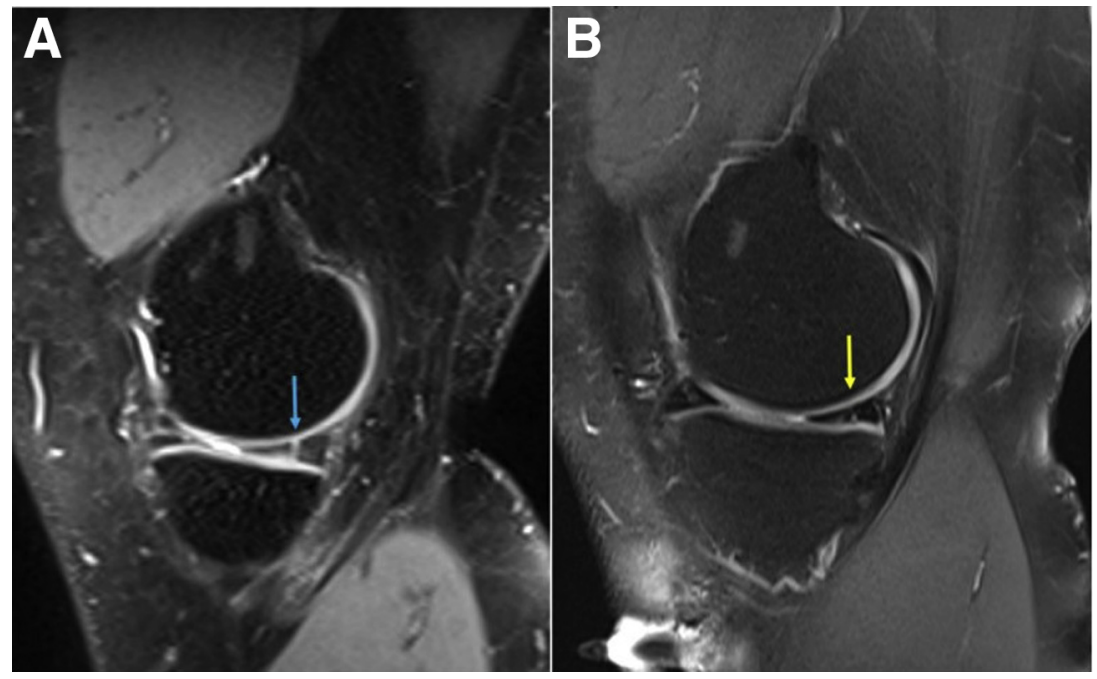

Fig 3. Proton density fat-suppressed sagittal magnetic resonance images without contrast in a 30-year-old patient with right knee trauma and an anterior cruciate ligament tear. (A) The preoperative sagittal scan shows a linear vertical fluid signal intensity (blue arrow) in the posterior horn of the medial meniscus consistent with a vertical tear. (B) The post-meniscal repair scan shows complete resolution of the vertical tear (yellow arrow).

intervals after meniscal repair. It is not always easy to differentiate between the high signal of fibrovascular and fibrocartilaginous repair tissue and the fluid signal of an unhealed tear (Figs 3 and 4). ${ }^{24,25}$ Almeida et al., ${ }^{26}$ using magnetic resonance arthrogram and computed tomography arthrogram, improved the rate of diagnosis of healing to $75 \%$ as opposed to $50 \%$ on conventional MRI. In an elegantly designed study, Yamasaki at al. ${ }^{27}$ compared conventional MRI and colored MRI T2 mapping of the repair site with the gold standard of second-look arthroscopy. Colored MRI T2 mapping accurately assessed the degree of healing at the repair site with high degrees of sensitivity and specificity. The high false-positive rate of $\mathrm{T} 2$-weighted images on conventional MRI must be borne in mind when counseling patients about the state of the repair and further planned interventions.

The time gap between injury and surgery is thought to influence meniscal repair healing. Tengrootenhuysen et al. ${ }^{9}$ reported greater success if repair was performed within 6 weeks of injury. However, Pujol et al. ${ }^{21}$ reported a failure rate of only $13 \%$ at 10 years in a population with an average time from injury to surgery of 114 months. Ahn et al. ${ }^{8}$ noted that

Fig 4. Proton density fat-suppressed sagittal magnetic resonance images without contrast in a 28-year-old patient with right knee trauma. (A) The preoperative sagittal scan shows a linear vertical and oblique fluid signal intensity (blue arrow) in the posterior horn of the medial meniscus consistent with a complex tear. (B) The post-meniscal repair scan shows increased fluid signal intensity (yellow arrow) at the site of repair, representing an unhealed tear.

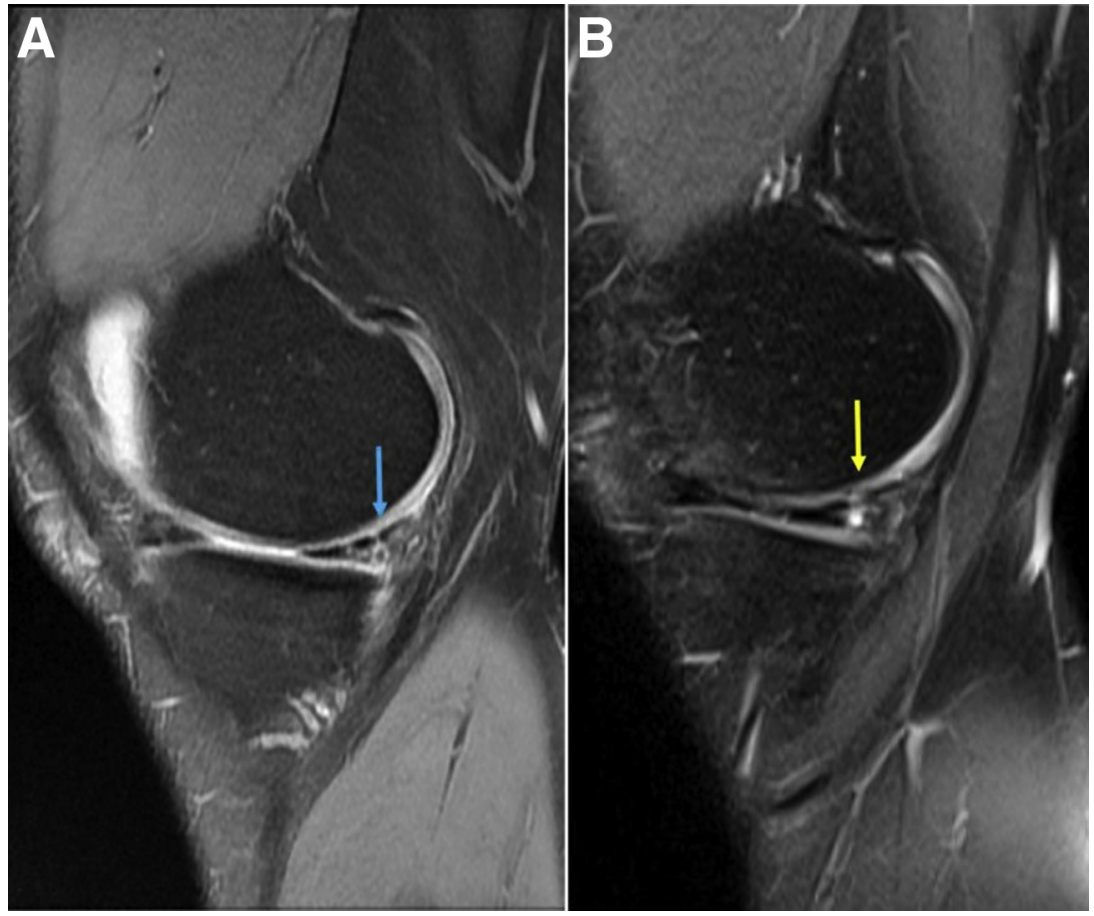


"location (red-red, red-white, or coexistence) was the only factor associated with complete healing of the meniscus." The vast injury-to-surgery time range of 3 days to 120 months in our small and heterogeneous group may be a factor in detecting any trends. ACLR in conjunction with meniscal repair is expected to have higher healing rates. Some reports have estimated a clinical success rate greater than $90 \%$ at 2 -year followup. ${ }^{28}$ Our study has shown better outcomes in patients who underwent ACLR compared with those who rejected ACLR in cases of concomitant ACL injury. Although this difference was not statistically significant, it does suggest that asymptomatic ACL injury may influence the meniscal repair outcome and, possibly, the long-term longevity of the knee.

Our patients' average KL grade was 2 compared with 1 in the contralateral knees. It is not possible to draw any firm conclusions from this finding, however. It must be noted that $84 \%$ of the knees that underwent radiography had an associated ACL injury, and 11 patients refused to undergo ACLR. It is well documented that combined ACL and meniscal injuries result in radiographic degenerative changes at 10 to 20 years after injury. ${ }^{29}$ Only $53.5 \%$ of our patients were able to return to their preinjury sporting level. Previous reports have shown a drop in the postoperative Tegner score even in professional athletes. ${ }^{20}$ The Tegner score drop from $6.3 \pm 1.1$ to $5.7 \pm 0.8$ in our patients is consistent with the conclusions of a systematic review by Eberbach et al. $^{30}$ assessing preinjury and postoperative Tegner scores.

\section{Limitations}

This study is limited by its retrospective nature, the heterogeneity and low number of patients, the lack of a control group, and the extremely wide time range between injury and surgery. In addition, $27.5 \%$ of the study patients underwent meniscal repair without ACLR of the ACL-deficient knee, which is a confounding factor to the study results.

\section{Conclusions}

The results of this study suggest that loaded deep knee flexion may be safe after a period of restricted rehabilitation, and clinical and radiologic tissue healing is independent of the overall functional outcome.

\section{References}

1. Netravali NA, Koo S, Giori NJ, Andriacchi TP. The effect of kinematic and kinetic changes on meniscal strains during gait. J Biomech Eng 2011;133:011006.

2. Allen PR, Denham RA, Swan AV. Late degenerative changes after meniscectomy. Factors affecting the knee after operation. J Bone Joint Surg Br 1984;66:666-671.

3. Lutz C, Dalmay F, Ehkirch FP, et al. Meniscectomy versus meniscal repair: 10 years radiological and clinical results in vertical lesions in stable knee. Orthop Traumatol Surg Res 2015;101:327-333.

4. Nepple JJ, Dunn WR, Wright RW. Meniscal repair outcomes at greater than five years: A systematic literature review and meta-analysis. J Bone Joint Surg Am 2012;94: 2222-2227.

5. Lee WQ, Gan JZ-W, Lie DTT. Save the meniscus-Clinical outcomes of meniscectomy versus meniscal repair. J Orthop Surg (Hong Kong) 2019;27:2309499019849813.

6. Ronnblad E, Barenius B, Engstrom B, Eriksson K. Predictive factors for failure of meniscal repair: A retrospective dual-center analysis of 918 consecutive cases. Orthop J Sports Med 2020;8:2325967120905529.

7. Uzun E, Misir A, Kizkapan TB, et al. Factors affecting the outcomes of arthroscopically repaired traumatic vertical longitudinal medial meniscal tears. Orthop J Sports Med 2017;5:2325967117712448.

8. Ahn JH, Lee YS, Yoo JC, et al. Clinical and second-look arthroscopic evaluation of repaired medial meniscus in anterior cruciate ligament-reconstructed knees. Am J Sports Med 2010;38:472-477.

9. Tengrootenhuysen M, Meermans G, Pittoors K, van Riet R, Victor J. Long-term outcome after meniscal repair. Knee Surg Sports Traumatol Arthrosc 201 1;19:236-241.

10. Richards DP, Barber FA, Herbert MA. Compressive loads in longitudinal lateral meniscus tears: A biomechanical study in porcine knees. Arthroscopy 2005:21: 1452-1456.

11. Spang RC III, Nasr MC, Mohamadi A, et al. Rehabilitation following meniscal repair: A systematic review. BMJ Open Sport Exerc Med 2018;4:000212.

12. Lin DL, Ruh SS, Jones HL, Karim A, Noble PC, McCulloch PC. Does high knee flexion cause separation of meniscal repairs? Am J Sports Med 2013;41:2143-2150.

13. Mulholland SJ, Wyss UP. Activities of daily living in nonWestern cultures: Range of motion requirements for hip and knee joint implants. Int J Rehabil Res 2001;24: 191-198.

14. Barrett GR, Field MH, Treacy SH, Ruff CG. Clinical results of meniscus repair in patients 40 years and older. Arthroscopy 1998; 14:824-829.

15. Collins NJ, Misra D, Felson DT, Crossley KM, Roos EM, Measures of knee function. Arthritis Care Res 2011;63: 208-228.

16. Kijowski R, Rosas H, Williams A, Liu F. MRI characteristics of torn and untorn post-operative menisci. Skeletal Radiol 2017;46:1353-1360.

17. Crues JV, Mink J, Levy TL, Lotysch M, Stoller DW. Meniscal tears of the knee: Accuracy of MR imaging. Radiology 1987; 164:445-448.

18. Kellgren JH, Lawrence JS. Radiological assessment of osteo-arthrosis. Ann Rheum Dis 1957;16:494-502.

19. Kang D-G, Park Y-J, Yu J-H, Oh J-B, Lee D-Y. A systematic review and meta-analysis of arthroscopic meniscus repair in young patients: Comparison of allinside and inside-out suture techniques. Knee Surg Relat Res 2019;31:1-11.

20. Devgan A, Magu NK, Siwach RC, Rohilla R, Sangwan SS. Functional outcome in athletes at five years of arthroscopic anterior cruciate ligament reconstruction. ISRN Orthop 2011;2011:570329. 
21. Pujol N, Tardy N, Boisrenoult P, Beaufils P. Long-term outcomes of all-inside meniscal repair. Knee Surg Sports Traumatol Arthrosc 2015;23:219-224.

22. Harput G, Guney-Deniz H, Nyland J, Kocabey Y. Postoperative rehabilitation and outcomes following arthroscopic isolated meniscus repairs: A systematic review. Phys Ther Sport 2020;45:76-85.

23. Miao Y, Yu JK, Zheng ZZ, et al. MRI signal changes in completely healed meniscus confirmed by second-look arthroscopy after meniscal repair with bioabsorbable arrows. Knee Surg Sports Traumatol Arthrosc 2009;17:622-630.

24. Arnoczky SP, Cooper TG, Stadelmaier DM, Hannafin JA. Magnetic resonance signals in healing menisci: An experimental study in dogs. Arthroscopy 1994;10:552-557.

25. Hoffelner T, Resch H, Forstner R, et al. Arthroscopic allinside meniscal repair-Does the meniscus heal? A clinical and radiological follow-up examination to verify meniscal healing using a 3-T MRI. Skeletal Radiol 201 1;40: 181-187.
26. Almeida AMD, Rodrigues MB, Rezende MUD, Pedrinelli A, Hernandez AJ. Meniscal repair results comparing MRI, arthro-MRI, and arthro-CT. Acta Ortop Bras 2018;26:166-169.

27. Yamasaki S, Hashimoto Y, Nishida Y, et al. Assessment of meniscal healing status by magnetic resonance imaging T2 mapping after meniscal repair. Am J Sports Med 2020;48: 853-860.

28. Toman CV, Dunn WR, Spindler KP, et al. Success of meniscal repair at anterior cruciate ligament reconstruction. Am J Sports Med 2009;37:1111-1115.

29. Lohmander LS, Englund PM, Dahl LL, Roos EM. The long-term consequence of anterior cruciate ligament and meniscus injuries: Osteoarthritis. Am J Sports Med 2007;35: 1756-1769.

30. Eberbach H, Zwingmann J, Hohloch L, et al. Sport-specific outcomes after isolated meniscal repair: A systematic review. Knee Surg Sports Traumatol Arthrosc 2018;26: 762-771. 